\title{
Classification of Image Database using SVM with Gabor Magnitude
}

\author{
Sultan Aljahdali \\ College of Computers \& Info Tech \\ Taif University, Taif, Saudi Arabia \\ aljahdali@tu.edu.sa
}

\author{
Aasif Ansari \\ College of Computers \& Info Tech \\ Taif University, Taif, Saudi Arabia \\ m.asif@tu.edu.sa
}

\author{
Nisar Hundewale \\ College of Computers \& Info Tech \\ Taif University, Taif, Saudi Arabia \\ n.hundewale@tu.edu.sa
}

\begin{abstract}
The CBIR term has been widely used to describe the process of retrieving desired images from a large collection on the basis of features (such as color, texture and shape) that can be extracted from the images themselves. In this paper, we have proposed an image retrieval system on the basis of classification using Support Vector Machine (SVM) which is implemented in MATLAB with the help of Gabor Filtered image features. In the proposed system, texture features are found by calculating the Standard Deviation of the Gabor Filtered image. A SVM classifier can be learned from training data of relevance images and irrelevance images marked by users. Using the classifier, the system can retrieve more images relevant to the query in the database efficiently. The proposed CBIR technique is implemented on a database having 1000 images spread across 11 categories and COIL image database having 1080 images spread across 15 categories. For each proposed CBIR technique 55 queries ( 5 per category) are fired on the database and net average precision and recall are computed. The results have shown performance improvement with higher precision and recall values, achieving crossover point as high as $89 \%$ with SVM technique as compared to image retrieval using Gabor Magnitude without SVM technique where the maximum crossover point is approximately $79 \%$.
\end{abstract}

\section{Keywords-CBIR, Gabor Magnitude, Support Vector Machine.}

\section{INTRODUCTION}

Databases of large images deal with the challenge of image indexing and retrieval[1],[2],[3],[4],[5] which has become one of the promising and important research area for researchers from a wide range of disciplines like computer vision, image processing and database areas. The thirst of better and faster image retrieval techniques is still appetizing to the researchers working in some of important applications for CBIR technology like art galleries [6],[7] museums, archaeology [8] architecture design [9],[10] geographic information systems [11] weather forecast [11],[12] medical imaging [11],[13] trademark databases [14],[15] criminal investigations [16],[17] image search on the Internet [18],[19],[20].

Researchers are discovering that the process of locating a desired image in a large and varied collection can be a source of considerable frustration [1],[15],[21]. Problems with traditional methods of image indexing [22],[23],[24] have led to the rise of interest in techniques for retrieving images on the basis of automatically-derived features such as color, texture and shape - a technology now generally referred to as Content-Based Image Retrieval (CBIR). After a decade of intensive research, CBIR technology is now beginning to move out of the laboratory and into the marketplace, in the form of commercial products like QBIC [25] and Virage [26]. However, the technology still lacks maturity, and is not yet being used on a significant scale [27]. In the absence of hard evidence on the effectiveness of CBIR in practice, opinion is still sharply divided about their usefulness in handling real-life queries in large and diverse image collections. Most of the current image indexing practices mainly rely on color, texture or shape features. The performance of the image retrieval technique improves if these features are used to train SVM and classify the image database based on trained SVM variables.

Automatic image annotation or image classification is an area of active research in the field of machine learning and pattern recognition. Retrieval systems have traditionally used manual image annotation for indexing and then later retrieving their image collections. However, manual image annotation is an expensive and labor intensive procedure [26]. Here, an automatic approach was proposed to categorize images based on a supervised learning technique. In supervised classification, collection of training images (labeled images) is given, and the problem is to label a newly encountered, yet unlabeled image. Each instance in the training set contains category or class specific labels and several image feature descriptors in the form of a combined feature vector.

In this paper, a hybrid approach to CBIR is taken. Initially, the Support Vector Machine (SVM) is to be trained and therefore the database of images is labeled using feedback from users which marks images as relevant or nonrelevant. The labeling of images has to be done only once to train SVM. This information is saved and the SVM is trained for the database. Once trained, SVM can subsequently be used to group together and classify images that may be semantically similar but quite distant in the lowlevel feature space. During training, a semantic feature space is constructed, and a query semantic feature vector is constructed for each semantic category.

In section 2 Theoretical considerations are elaborated; section 3 deals with feature vector generation algorithm. Proposed CBIR method is given in section 4, results and discussion are given in section 5 and conclusions are presented in section 6 .

\section{THEORITICAL CONSIDERATIONS}

\section{A. Content Based Image Retrieval}

The earliest use of the term content-based image retrieval in the literature seems to have been by Kato et.al.[28] to describe his experiments into automatic retrieval of images from a database by color and shape 
feature. The term has since been widely used to describe the process of retrieving desired images from a large collection on the basis of features (such as color, texture and shape) that can be automatically extracted from images themselves. The typical CBIR system performs two major tasks [1], [15]. The first one is feature extraction (FE), where a set of features, called image signature or feature vector, is generated to accurately represent the content of each image in the database. A feature vector is much smaller in size than the original image, typically of the order of hundreds of elements (rather than millions). The second task of CBIR system is similarity measurement (SM) Here a distance between the query image and each image in the database using their signatures is computed so that the top "closest" images can be retrieved. Non-transform based image coding can have image features such as color more easily available. For example, recent work on color image coding using vector quantization [29],[30],[31] has demonstrated that, color as well as pattern information can be readily available in the compressed image stream (without performing decoding) to be used as image indices for effective and efficient image retrieval (i,e image indexing using a colored pattern appearance model.)

\section{B. Support Vector Machine}

Support vector machine (SVM) has been a promising method for data classification and regression [32], [33], [34], [35], [36]. Its success in practice is drawn by its solid mathematical foundation which conveys the following two salient properties:

Margin maximization: The classification boundary function of SVM maximizes the margin, which in machine learning theory, corresponds to maximizing the generalization performance given a set of training data.

Nonlinear transformation of the feature space using the kernel trick: SVM handles a nonlinear classification efficiently using the kernel trick which implicitly transforms the input space into another high dimensional feature space.

Support vector machines (SVMs) are a set of related supervised learning methods used for classification and regression [38]. They belong to a family of generalized linear classifiers. In another terms, Support Vector Machine (SVM) is a classification and regression prediction tool that uses machine learning theory to maximize predictive accuracy while automatically avoiding over-fit to the data. Support Vector machines can be defined as systems which use hypothesis space of a linear functions in a high dimensional feature space, trained with a learning algorithm from optimization theory that implements a learning bias derived from statistical learning theory.

SVM is a useful technique for data classification. Even though it's considered that Neural Networks are easier to use than this, however, sometimes unsatisfactory results are obtained. A classification task usually involves with training and testing data which consist of some data instances [39]. Each instance in the training set contains one target values and several attributes. The goal of SVM is to produce a model which predicts target value of data instances in the testing set which are given only the attributes [33].

Classification in SVM is an example of Supervised Learning. Known labels help indicate whether the system is performing in a right way or not. This information points to a desired response, validating the accuracy of the system, or be used to help the system learn to act correctly. A step in SVM classification involves identification as which are intimately connected to the known classes. This is called feature selection or feature extraction. Feature selection and SVM classification together have a use even when prediction of unknown samples is not necessary. They can be used to identify key sets which are involved in whatever processes distinguish the classes [33].

\section{Similarity Measure}

Finding good similarity measures between images based on some feature set is a challenging task. On the one hand, the ultimate goal is to define similarity functions that match with human perception, but how humans judge the similarity between images is a topic of ongoing research. Many Current Retrieval systems take a simple approach by using typically norm-based distances (e.g., Euclidean distance [9]) on the extracted feature set as a similarity function. The main premise behind these CBIR systems is that given a "good set" of features extracted from the images in the database (the ones that significantly capture the content of images.) then for two images to be "similar" their extracted features have to be "close" to each other.

The Direct Euclidian Distance between an image P and query image $\mathrm{Q}$ can be given as the equation below.

$$
E D=\sqrt{\sum_{i=1}^{n}\left(v_{p i}-v_{q i}\right)^{2}}
$$

Where, Vpi and Vqi are the feature vectors of image P and Query image $Q$ respectively with size ' $n$ '. Beside the Euclidean Distance, there are many ways to measure feature distance between two images for example: Manhattan distance; the Mahalanobis Distance; Earth Mover's Distance (EMD) and the chord distance [15],[24].

\section{FEATURE VECTOR EXTRACTION}

\section{Gabor Magnitude}

Gabor filters are a group of wavelets, with each wavelet capturing energy at a specific frequency and a specific direction. Expanding a signal using this basis provides a localized frequency description, therefore capturing local features/energy of the signal. Texture features can then be extracted from this group of energy distributions. The scale (frequency) and orientation tunable property of Gabor filter makes it especially useful for texture analysis. The filters of a Gabor filter bank are designed to detect different frequencies and orientations. We use them to extract features on key points detected by interest operators 
[41].From each filtered image, Gabor features can be calculated and used to retrieve images. The procedure for extracting the Gabor feature vector is shown in Figure 1. The algorithm (figure 2) and equations (2-5) related to extraction of Gabor features are also mentioned below figure1 [37],[40].

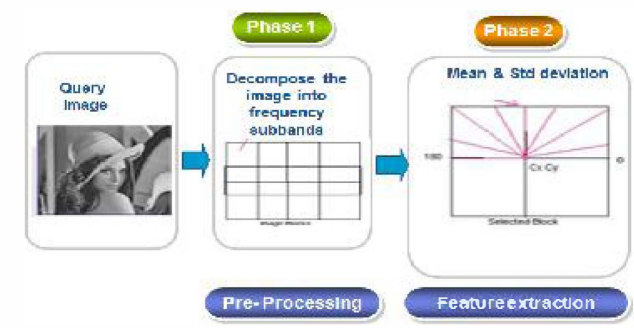

Figure 1. Gabor Filter Feature Extraction.

For a given image $\mathrm{I}(\mathrm{x}, \mathrm{y})$, the discrete Gabor wavelet transform is given by a convolution:

$$
W_{m n}=\sum_{x_{1}} \sum_{y_{1}} I\left(x_{1}, y_{1}\right) g_{m n} *\left(x-x_{1}, y-y_{1}\right)
$$

where $*$ indicates complex conjugate and where $\mathrm{m}, \mathrm{n}$ specify the scale and orientations of wavelet respectively.

After applying Gabor filters on the image with different orientation at different scale, we obtain an array of magnitudes:

$$
E(m, n)=\sum_{x} \sum_{y}\left|W_{m n}(x, y)\right|
$$

These magnitudes represent the energy content at different scale and orientation of the image. The main purpose of texture-based retrieval is to find images or regions with similar texture.

The standard deviation $\sigma$ of the magnitude of the transformed coefficients is:

$\sigma_{m n}=\sqrt{\frac{\sum_{x} \sum_{y}\left(\left|W_{m n}(x, y)\right|-\mu_{m n}\right)^{2}}{P \times Q}}$

where $\mu_{m n}=\frac{E(m, n)}{P \times Q}$ is the mean of magnitude

A feature vector $\mathbf{f}$ (texture representation) is created using $m n$ and $m n$ as the feature components [25], [42]. $M$ scales and $N$ orientations are used and the feature vector is given by:

$f=\left[\sigma_{00}, \sigma_{01} \ldots, \sigma_{(M-1)(N-1)}\right]$

$f_{\text {Gabor }}=\frac{f-\mu}{\sigma}$ where $\mu$ is the mean and $\sigma$ is the standard deviation of $f$.The basic algorithm for Gabor feature extraction is shown below:

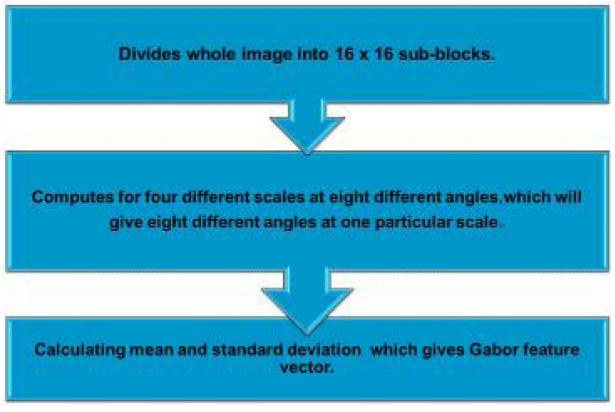

Figure 2. Gabor Filter Algorithm.

\section{PROPOSED CBIR TECHNIQUE}

1. We consider a database created by transferring the images in the hard disk. These images will be forwarded to Feature Vector Module i.e. Gabor Filter.

2. Gabor filter which is basically used for shape and texture features, it will first scale the original image or query image into the size of $256 \times 256$ pixels. After scaling, the image is divided into equal blocks each of the size $16 \times 16$ pixels [44][45].

3. By applying Gabor Filter with different orientations and different scales on all the blocks of an image, we obtain a set of magnitudes for each block. We compare the standard deviation for each orientation and obtain Gabor feature for that block and subsequently a feature vector is obtained for the entire image [47]. Thus, features are calculated for each and every image and the resulted feature vectors are stored in feature vector database [43].

4. Label the feature vectors of images from all the classes (training).

5. Train SVM according to the label attached to the feature vectors.

6. Label the query image with the class it belongs.

7. Extract variables of the trained SVM for required class.

8. Perform classification of the images.

9. The indexed images are stored in the result folder.

\section{IMPLEMENTATION}

In this approach Gabor feature vector is obtained by calculating Standard Deviation of Gabor. The SVM is trained by labeling the feature vectors of the known images. The variables generated while training the SVM are then used for the purpose of classification and hence the images are retrieved.

\section{A. Image databse}

The proposed CBIR technique is implemented in MATLAB on a generic database [46] having 1000 images spread across 11 categories and COIL image database [48] 
having 1080 images spread across 15 categories. For each proposed CBIR technique 55 queries ( 5 per category) are fired on the database and net average precision and recall are computed. The distribution of the images of generic database is shown in Table 1 [37].

TABLE 1:Generic Databases

\begin{tabular}{lc}
\multicolumn{2}{c}{ DABLE 1:Generic Databases } \\
\hline Category & Number Of Images \\
\hline Tribal People & 85 \\
Buses & 99 \\
Beaches & 99 \\
Dinosaurs & 99 \\
Elephants & 99 \\
Roses & 99 \\
Horses & 99 \\
Snow Mountains & 61 \\
Food & 100 \\
Historical Monuments & 99 \\
Sunrise Scenery & 61 \\
\hline
\end{tabular}

To compare the techniques and to check their performance we have used the precision and recall.

\section{B. Retrieval accuracy}

To assess the retrieval effectiveness, we have used the precision and recall as statistical comparison parameters for the Gabor Filter [49].

It is given by

$$
\begin{aligned}
& \text { Precision }=\frac{\text { Number of relevant images retrieved }}{\text { Total number of images retrieved }} \\
& \text { Recall }=\text { Total number of relevant images retrieved in } \mathrm{db} b
\end{aligned}
$$

\section{RESULTS AND DISCUSSION}

The methods SVM-Gabor Magnitude and only Gabor Magnitude Features were applied to the image database having 1000 images spread across 11 categories and COIL image database having 1080 images spread across 15 categories. The query and database image matching is done using SVM classifier. The average precision and average recall are computed by grouping the number of retrieved images sorted according to classification of database images with the query image.

\section{A. Generic database}

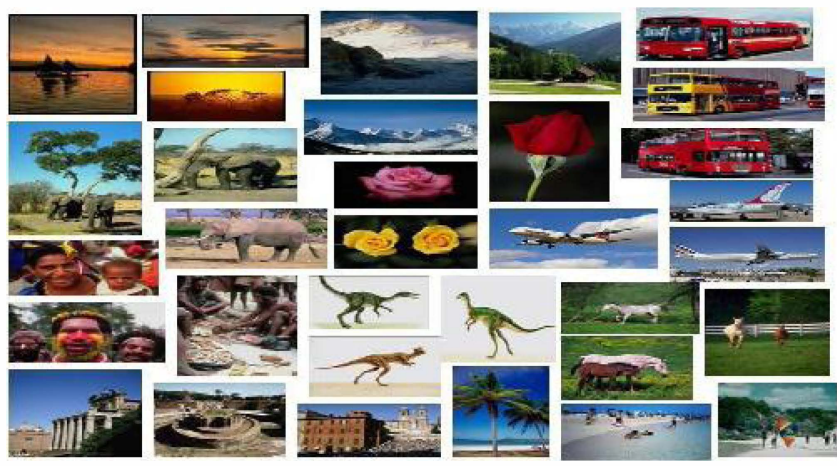

Figure 3. Sample images of Generic Image Database [Image database contains total 1000 images with 11 categories].

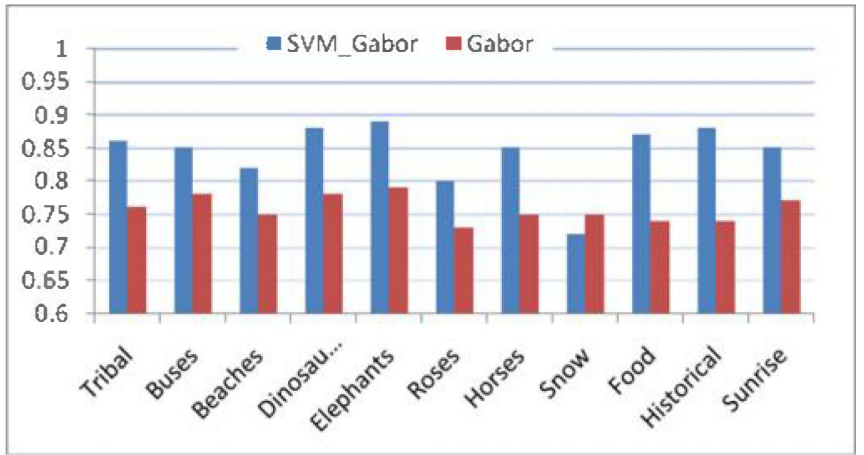

Figure 4. Crossover points for Precision \& Recall plotted against Number of retrieved images for different image Categories on Generic Database.

Figure 4 shows the graphs of precision/recall values plotted against number of retrieved images for all proposed image retrieval techniques. Here SVM -Gabor based image retrieval technique gives the highest precision/recall crossover values specifying the best performance. The crossover point varies

for different image category, and the highest crossover point 0.89 is achieved for Elephants and Dinosaurs image category.

Fig. 5(a) below shows results obtained using Gabor Filtered image features based CBIR technique and Fig. 5(b) shows results obtained using SVM-Gabor based CBIR technique. There is a significant improvement in results using SVM based technique. The crossover point for the SVM-Gabor based CBIR technique is 0.89 . whereas the crossover point is 0.79 for the results obtained when using Gabor filtered image features based CBIR technique alone. 


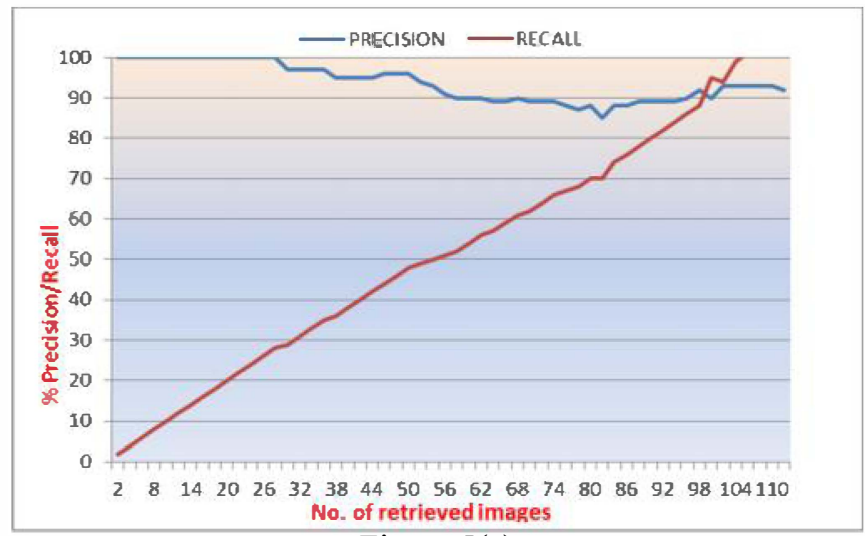

Figure 5(a)

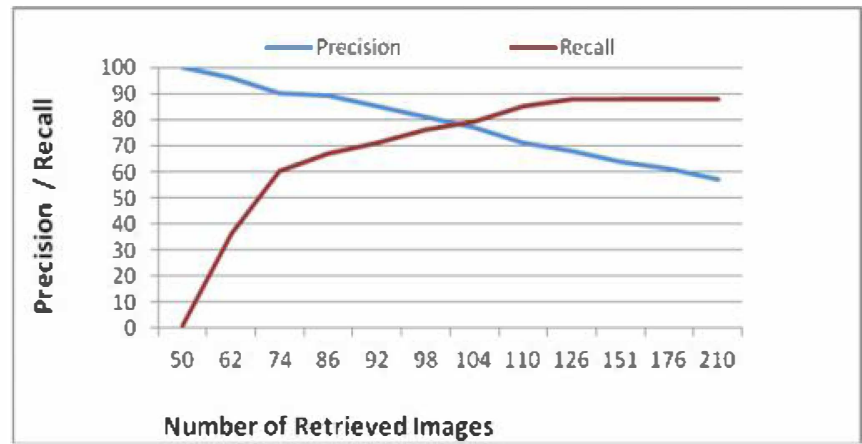

Figure 5(b)

Precision \& Recall plotted against for SVM-Gabor based CBIR technique on Generic database is shown in Figure 5(a). Whereas Figure 5(b) shows Precision \& Recall plotted against for Gabor Filtered image features based CBIR technique on Generic database.

The precision/recall values and crossover points proves that the discrimination capability of SVM - Gabor Magnitude based CBIR technique is better than Gabor Filtered image features based CBIR technique.

However the distinction in the performance of all these techniques is not very clear. The height of crossover point of precision and recall curves plays very important role in performance comparison of CBIR methods. Ideally this crossover point height should be one. Higher the value of this crossover point better the performance is.

\section{B. COIL database}
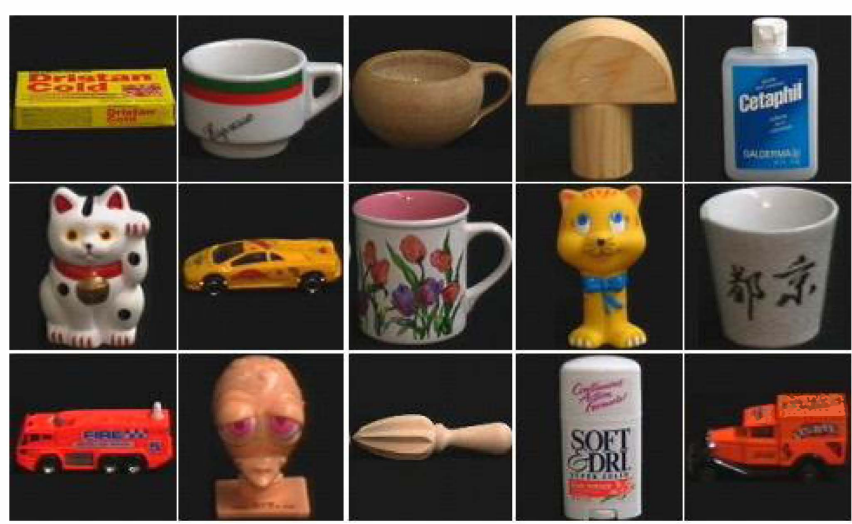

Figure 6. Sample of 15 images of COIL image database the database has 15 categories, for a total of 1,080 images.

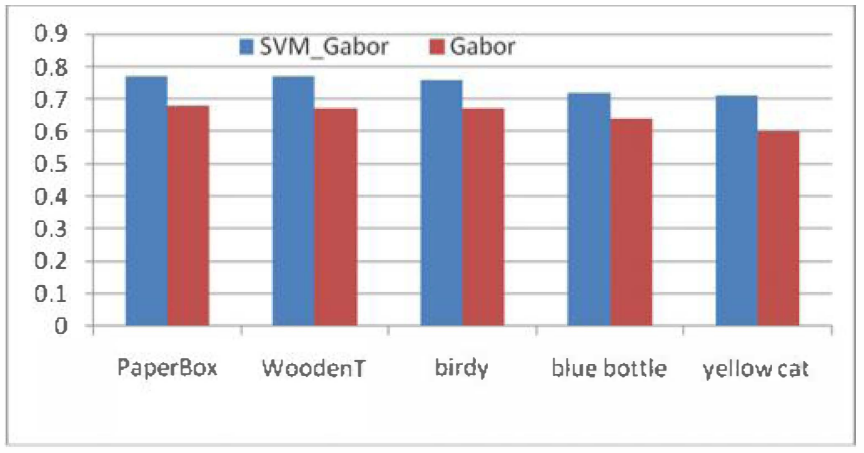

Figure 7. Crossover points for Precision \& Recall plotted against Number of retrieve images for different Image Categories on Coil Database.

Figure 7 shows the graphs of precision/recall values plotted against number of retrieved images for all proposed image retrieval techniques. Here SVM-Gabor based image retrieval technique gives the highest precision/recall crossover values specifying the best performance. The crossover point varies for different image category, and the highest crossover point 0.78 is achieved for Paper Box and Wooden $\mathrm{T}$ image category.

Figure 8(a) below shows results obtained using SVM-Gabor based technique tested on COIL image database. There is significant improvement in results using SVM-Gabor technique. The precision-recall curve shows crossover point of $77 \%$ i.e., 0.77 whereas, Figure 8(b) shows results obtained using only Gabor based CBIR technique. The results degrade slightly as number of images go on increasing. The crossover point for the Gabor based CBIR technique is 0.68 . 


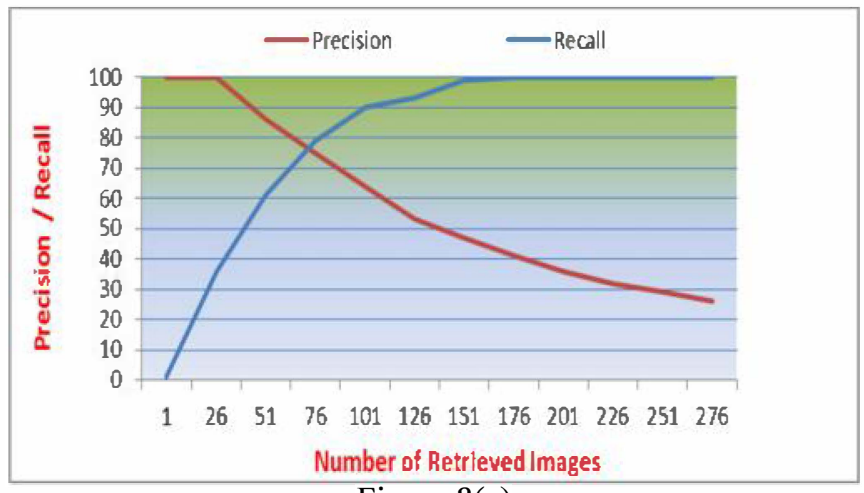

Figure 8(a)

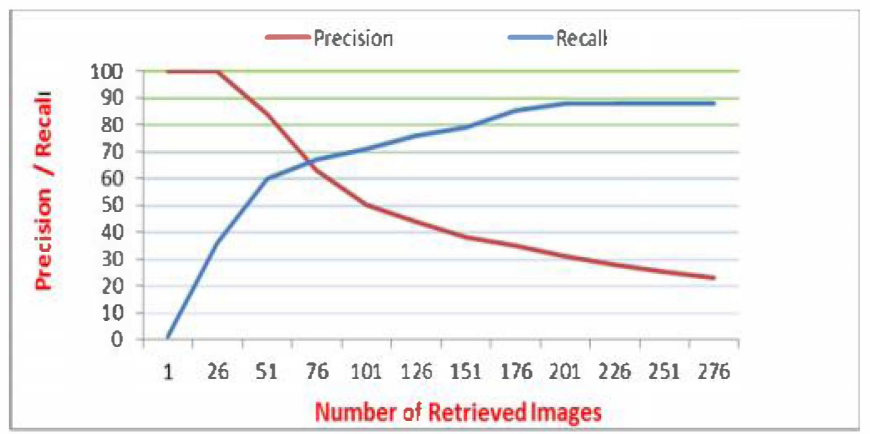

Figure 8(b)

Figure 8(a) shows Precision \& Recall plotted against for SVM-Gabor based CBIR technique on COIL database and Figure 8(b) shows Precision \& Recall plotted against for Gabor based CBIR technique on COIL database.

\section{CONCLUSION}

In this paper we have proposed a Content Based Image Retrieval System using Support Vector Machine based on Gabor Filter's Response of an image. The proposed system is giving higher Precision and Recall as compared to the CBIR technique when only Gabor magnitude features are used. The superiority of the system is because of the Gabor feature gives good response to texture of the image and makes it very clear for SVM to classify and retrieve the required image.

\section{REFERENCES}

[1] H.B.Kekre, Sudeep D. Thepade, "Image Retrieval using Augmented Block Truncation Coding Techniques", ACM International Conference on Advances in Computing, Communication and Control (ICAC3-2009), pp. 384-390, 23-24 Jan 2009, Fr.ConceicaoRodrigous College of Engg., Mumbai. Is uploaded on online ACM portal.

[2] Minh N. Do, Martin Vetterli, "Wavelet-Based Texture Retrieval Using Generalized Gaussian Density and Kullback-Leibler Distance", IEEE Transactions On Image Processing, Volume 11, Number 2, pp.146-158, February 2002.

[3] B.G.Prasad, K.K. Biswas, and S. K. Gupta, "Region -based image retrieval using integrated color, shape, and location index", International Journal on Computer Vision and Image
Understanding Special Issue: Colour for Image Indexing and Retrieval,Volume 94, Issues 1-3, April-June 2004, pp.193-233.

[4] H.B.Kekre, Tanuja Sarode, Sudeep D. Thepade, "DCT Applied to Row Mean and Column Vectors in Fingerprint Identification", In Proceedings of International Conference on Computer Networks and Security (ICCNS), 27-28 Sept. 2008, VIT, Pune.

[5] Zhibin Pan, Kotani K., Ohmi T., "Enhanced fast encoding method for vector quantization by finding an optimally-ordered Walsh transform kernel", ICIP 2005, IEEE International Conference, Volume 1, pp I - 573-6, Sept. 2005.

[6] H.B.kekre, Sudeep D. Thepade, "Improving 'Color to Gray and Back' using Kekre's LUV Color Space”, IEEE International Advanced Computing Conference 2009 (IACC'09), Thapar University, Patiala, INDIA, 6-7 March 2009. Is uploaded and available online at IEEE Xplore.

[7] H.B.Kekre, Sudeep D. Thepade, "Color Traits Transfer to Grayscale Images", In Proc.of IEEE First International Conference on Emerging Trends in Engg. \& Technology, (ICETET-08), G.H.Raisoni COE, Nagpur, INDIA. Uploaded on online IEEE Xplore.

[8] H.B.Kekre, Sudeep D. Thepade, "Scaling Invariant Fusion of Image Pieces in Panorama Making and Novel Image Blending Technique", International Journal on Imaging (IJI), www.ceser.res.in/iji.html, Volume 1, No. A08, pp. 31-46, Autumn 2008.

[9] H.B.Kekre, Sudeep D. Thepade, "Creating the Color Panoramic View using Medley of Grayscale and Color Partial Images", WASET International Journal of Electrical, Computer and System Engineering (IJECSE), Volume 2, No. 3, Summer 2008.

[10] H.B.Kekre, Sudeep D. Thepade, "Image Blending in Vista Creation using Kekre's LUV Color Space", SPIT-IEEE Colloquium and International Conference, Sardar Patel Institute of Technology, Andheri, Mumbai, 04-05 Feb 2008.

[11] H.B.Kekre, Sudeep D. Thepade, "Rendering Futuristic Image Retrieval System", National Conference on Enhancements in Computer, Communication and Information Technology, EC2IT-2009, 20-21 Mar 2009, K.J.Somaiya College of Engineering, Vidyavihar, Mumbai-77.

[12] H.B.Kekre, Sudeep D. Thepade, "Image Retrieval using NonInvolutional Orthogonal Kekre's Transform", International Journal of Multidisciplinary Research and Advances in Engineering (IJMRAE), Ascent Publication House, 2009, Volume 1, No.I, pp 189-203, 2009. Abstract available online at www.ascent-journals.com (ISSN: 0975-7074)

[13] H.B.Kekre, Sudeep D. Thepade, ArchanaAthawale, Anant Shah, PrathmeshVerlekar, SurajShirke,"Walsh Transform over Row Mean and Column Mean using Image Fragmentation and Energy Compaction for Image Retrieval", International Journal on Computer Science and Engineering (IJCSE),Volume 2S, Issue 1, January 2010, (ISSN: 0975-3397).

[14] H.B.Kekre, Tanuja Sarode, Sudeep D. Thepade, "Color-Texture Feature based Image Retrieval using DCT applied on Kekre's Median Codebook", International Journal on Imaging (IJI), Volume 2, Number A09, Autumn 2009,pp. 55-65. Available online at www.ceser.res.in/iji.html (ISSN: 0974-0627).

[15] H.B.Kekre, Sudeep D. Thepade, "Boosting Block Truncation Coding using Kekre's LUV Color Space for Image Retrieval", WASET International Journal of Electrical, Computer and System Engineering (IJECSE), Volume 2, Number 3, pp. 172180, Summer 2008.

[16] H.B.Kekre, Sudeep D. Thepade, Archana Athawale, Anant Shah, Prathmesh Verlekar, Suraj Shirke, "Performance Evaluation of Image Retrieval using Energy Compaction and Image Tiling over DCT Row Mean and DCT Column Mean", Springer-International Conference on Contours of Computing Technology (Thinkquest-2010), BabasahebGawde Institute of Technology, Mumbai, 13-14 March 2010, The paper will be uploaded on online Springerlink. 
[17] H.B.Kekre, Tanuja K. Sarode, Sudeep D. Thepade, VaishaliSuryavanshi,"Improved Texture Feature Based Image Retrieval using Kekre's Fast Codebook Generation Algorithm", Springer-International Conference on Contours of Computing Technology (Thinkquest-2010), BabasahebGawde Institute of Technology, Mumbai, 13-14 March 2010, The paper will be uploaded on online Springerlink.

[18] Stian Edvardsen, "Classification of Images using color, CBIR Distance Measures and Genetic Programming", Ph.D. Thesis, Master of science in Informatics, Norwegian university of science and Technology, Department of computer and Information science, June 2006

[19] H.B.Kekre, Sudeep D. Thepade, "Image Retrieval using ColorTexture Features Extracted from Walshlet Pyramid", ICGST International Journal on Graphics, Vision and Image Processing (GVIP), Volume 10, Issue I, Feb.2010, pp.9-18.

[20] Gudivada V N and Raghavan V V "Design andvevaluation of algorithms for image retrieval by spatial similarity" ACM Trans. on Information Systems, Vol. 13, Num. 2, pp 115-144, 1995.

[21] H.B.Kekre, Sudeep D. Thepade, "Rendering Futuristic Image Retrieval System", In Proc. Of National Conference EC2IT2009, KJSCOE, Mumbai, 20-21 Mar 2009.

[22] Y. Rui, T. S. Huang, S. Mehrotra, M. Ortega, "Automatic matching tool selection using relevance feedback in MARS", In Proc. of Int. Conf. on Visual Information Systems, pp 109-116, San Diego, CA,Dec. 1997

[23] M. La Cascia, S. Sethi, S. Sclaroff. "Combining textual and visual cues for content-based image retrieval on the world wide web", In IEEE Workshop on Content-based Access of Image and Video Libraries, pp 24-28, Santa Barbara, CA, June 1998

[24] H.B.Kekre, Sudeep D. Thepade, "Using YUV Color Space to Hoist the Performance of Block Truncation Coding for Image Retrieval”, IEEE International Advanced Computing Conference 2009 (IACC'09), Thapar University, Patiala, INDIA, 6-7 March 2009.

[25] Flickner M. et al, "Query by image and video content: the QBIC system", IEEE Computer 1995, Volume 28, Number 9, pp 2332.

[26] Gupta A. et al, "The Virage image search engine: an open framework for image management", in Storage and Retrieval for Image and Video Databases IV, Proc SPIE 2670, 1996, pp 76-87.

[27] Gudivada V N and Raghavan V V "Design and evaluation of algorithms for image retrieval by spatial similarity" ACM Trans. on Information Systems, Vol. 13, Num. 2, pp 115-144, 1995.

[28] Hirata K. and Kato T. "Query by visual example content-based image retrieval", In Proc. of Third International Conference on Extending Database Technology, EDBT'92, 1992, pp 56-71

[29] Zhibin P.; Kotani, K.; Ohmi, T.,"Enhanced fast encoding method for vector quantization by finding an optimally-ordered Walsh transform kernel", ICIP05, IEEE Int. Conf., Vol.1, pp I573, Sept. 2005.

[30] H. B. Kekre, Tanuja K. Sarode, "Fast Codebook Search Algorithm for Vector Quantization using Sorting Technique", ACM Int. Conf. ICAC3-2009, 23-24 Jan 2009, FCRCE, Mumbai. Available on ACM portal.

[31] H. B. Kekre, Tanuja K. Sarode, Bhakti Raul, "Color Image Segmentation using Kekre's Fast Codebook Generation Algorithm Based on Energy Ordering Concept", ACM Int. Conference ICAC3-2009, 23- 24 Jan 2009, FCRCE, Mumbai. Available on ACM portal

[32] V. N. Vapnik, "Statistical Learning Theory" John Wiley and Sons, 1998.

[33] C. J. C. Burges, "A tutorial on support vector machines for pattern recognition" Data Mining and Knowledge Discovery, 2:121-167, 1998.

[34] T. Joachims, "Text categorization with support vector machines" In Proc. 10th European Conference on Machine
Learning (ECML'98), pages 137-142, Chemnitz, Germany, 1998.

[35] C.-C. Chang and C.-J. Lin., "Training nu-support vector classifiers: Thoery and algorithms" Neural Computation, 13:2119-2147, 2001

[36] H. Yu, J. Han, and K. C. Chang., "PEBL: Positive-example based learning for Web page classification using SVM" In Proc 8th Int. Conf. Knowledge Discovery and Data Mining (KDD’02), pages 239-248, Edmonton, Canada, 2002.

[37] Sinora Banker Ghosalkar, Vinayak A.Bharadi, Sanjay Sharma, Asif Ansari, "Feature Extraction using Overlap Blocks for Content based Image Retreival" International Journal of Computer Applications (0975-8887), Volume 28-No.7, August 2011.

[38] Duda R. and Hart P., "Pattern Classification and Scene Analysis", Wiley, New York 1973

[39] Nello Cristianini and John Shawe-Taylor, "An Introduction to Support Vector Machines and Other Kernel-based Learning Methods", Cambridge University Press, 2000.

[40] H.B. Kekre, V.A. Bharadi, S.D. Thepade, B.K. Mishra, S.E Ghosalkar, S.M. Sawant, "Content Based Image Retreival Using Fusion of Gabor Magnitude and Modified Block Truncation Coding," icetet, pp.140-145, 2010 3rd International Conference on Emerging Trends in Engineering and Technology, 2010.

[41] ArtiKhaparde,,B.L.Deekshatulu,M.Madhavilath,ZakiraFarheen, Sandhya Kumari V, "Content Based Image Retrieval Using Independent Component Analysis", IJCSNS International Journal ofComputer Science and Network Security, VOL.8 No.4, April 2000

[42] Faloutsos, C et al (1994) "Efficient and effective querying by image content" Journal of Intelligent Information Systems 3, 231-262.

[43] Dr.H.B.Kekre, Ms. Swapna Borde," Content Based Image Retrieval," National Conference on Applications of Digital Signal Processing,NCDSP-2007, January 19-20, 2007.

[44] Lei Zhu, Chun Tang, Aibing Rao and Aidong Zhang,"Using Thesaurus To Model Keyblock-Based Image Retrieval ," Technical Report, Department of Computer Science and Engineering , State University of New York At Buffalo, Buffalo,NY 14260,USA.

[45] Dengsheng Zhang, Aylwin Wong, Maria Indrawan, Guojun Lu ,"Content-based Image Retrieval Using Gabor Texture Features"Gippsland School of Computing and Information Technology.

[46] http://wang.ist.psu.edu/ jwang/test1.tar

[47] X. Fu, Y. Li, R. Harrison, S. Belkasim, "Content-based Image Retrieval Using Gabor-Zernike Features", The 18th International Conference on Pattern Recognition (ICPR'06).

[48] http://www.cs.columbia.edu/CAVE/software/softlib/coil100.php

[49] Rajashekhara," Novel Image Retrieval Techniques: domain specific approaches," Ph.D. Thesis Department of Electrical Engineering Indian Institute of Technology - Bombay, 2006. 